\title{
Síntese do Polipirrol com Surfactantes Aniônicos Visando Aplicações como Absorvedores de Micro-Ondas
}

\author{
Regiane Aparecida Medeiros Campos \\ Instituto Tecnológico da Aeronáutica - ITA \\ Roselena Faez \\ Laboratório de Materiais Poliméricos e Biossorventes, Departamento de Ciências da Natureza, \\ Matemática e Educação, Universidade Federal de São Carlos \\ Mirabel Cerqueira Rezende \\ Instituto de Ciência e Tecnologia/UNIFESP, São José dos Campos. \\ Instituto Tecnológico da Aeronáutica - ITA
}

\begin{abstract}
Resumo: Neste trabalho diferentes amostras de polipirrol (PPi) foram sintetizadas variando a combinação dos oxidantes $\mathrm{FeCl}_{3}$ e $\mathrm{Fe}_{2}\left(\mathrm{SO}_{4}\right)_{3}$ e dos surfactantes docecilbenzenosulfonato de sódio (DBSNa) e ácido dodecilbenzenosulfônico (DBSA). Análises por espectroscopia na região do infravermelho não apresentam diferenças significativas na estrutura química das amostras de PPi sintetizadas. No entanto, análises termogravimétricas, de condutividade elétrica e de difração de raios X mostram que os surfactantes aniônicos favorecem a obtenção de amostras mais estáveis termicamente, condutoras e ordenadas cristalograficamente. Medidas de refletividade de blendas de PPi com resina epóxi, na faixa de 8 a $12 \mathrm{GHz}$, mostram valores de atenuação de micro-ondas de até 95\% da radiação incidente.
\end{abstract}

Palavras-chave: Polipirrol, absorvedores de micro-ondas, síntese.

\section{Synthesis of Polypyrrole with Anionic Surfactants Targeting Applications such as Microwave Absorbers}

\begin{abstract}
In this work different samples of polypyrrole (PPy) were synthesized varying the combination of $\mathrm{FeCl}_{3}$ and $\mathrm{Fe}_{2}\left(\mathrm{SO}_{4}\right)_{3}$ as oxidant agents and sodium dodecylbenzenesulfonate (DBSNa) and dodecylbenzenesulfonic acid (DBSA) as surfactants. Infrared spectroscopy analyses showed no significant differences in the chemical structures of synthesized PPy samples. However, thermogravimetric, electrical conductivity and X-ray diffraction analyses showed that the anionic surfactants favored the preparation of samples which are more thermally stable, conducting and with a more ordered crystallographic structure. Reflectivity measurements of PPy/epoxy resin blends, in the frequency range from 8 to $12 \mathrm{GHz}$, showed microwave attenuation up to $95 \%$ of the incident radiation.
\end{abstract}

Keywords: Polypyrrole, radar absorbing materials, synthesis.

\section{Introdução}

Com os acelerados desenvolvimentos tecnológico e industrial nas áreas da eletrônica e, principalmente, de telecomunicações, os mais diversos tipos de produtos eletrônicos, como equipamentos de entretenimento, dispositivos eletrônicos portáteis, computadores, celulares e antenas de transmissão de sinais na faixa de micro-ondas, estão cada vez mais difundidos e presentes na vida cotidiana ${ }^{[1,2]}$. Esses equipamentos utilizam os mais diversos tipos de materiais, com propriedades elétricas que vão desde isolantes a condutores, abrangendo praticamente todo o espectro de condutividade elétrica.

Um histórico sobre a tecnologia de polímeros evidencia, sem dúvida alguma, que uma das propriedades mais importantes desses materiais sintéticos é a possibilidade de se comportarem como excelentes isolantes elétricos, tanto em altas frequências (de $\mathrm{Hz}$ a $\mathrm{GHz}$ ), quanto em voltagens elevadas. No entanto, a classe dos polímeros intrinsecamente condutores (PIC) vem sendo extensivamente estudada, devido, principalmente, à sua característica de conduzir eletricidade, pois sua estrutura química é formada por longos sistemas $\pi$ conjugados.

O polipirrol (PPi), um dos PIC mais estudados, é conhecido desde 1968 quando Dall'Olio et al. ${ }^{[3]}$ obteve um pó preto aderido à superfície do eletrodo ao eletrolisar uma solução de pirrol em ácido sulfúrico. As cadeias poliméricas do PPi são formadas de unidades aromáticas, ligadas por meio de átomos de carbono ${ }^{[4]}$. Esse é um dos polímeros condutores mais promissores, juntamente com a polianilina, para trabalhos de pesquisa e desenvolvimento em diversas áreas, devido à sua estabilidade química, facilidade de síntese e elevada condutividade elétrica. Este polímero passou a receber maior atenção a partir de 1979 , quando Diaz et al. ${ }^{[5]}$ obtiveram um filme preto de PPi, a partir da eletrólise de uma solução de pirrol em acetonitrila e tetrafluorborato de tetrametilamônio. 
O PPi, bem como outros polímeros condutores, pode ser sintetizado via rotas química e eletroquímica. A rota química leva à formação de um pó preto quando uma solução de pirrol entra em contato com uma solução de um agente oxidante, por exemplo, o $\mathrm{FeCl}_{3}{ }^{[6]}$. $\mathrm{O}$ uso de surfactantes é, também, comum na síntese de PPi e pode influenciar a preparação do PPi de três principais formas: (1) os surfactantes aniônicos podem formar ligação iônica com o policátion PPi; (2) a parte hidrofóbica das moléculas do surfactante pode adsorver o polímero condutor produzido, e (3) micelas surfactantes, se presentes, podem afetar a distribuição dos reagentes entre as fases micelar e aquosa, alterando o curso da reação de polimerização do pirrol $^{[7]}$.

Por apresentarem condutividade elétrica, os polímeros condutores vêm sendo estudados no processamento de materiais absorvedores de radiação eletromagnética (MARE), pelo fato desses materiais favorecerem a troca de energia da onda eletromagnética, na faixa de microondas, por energia térmica, atenuando, assim, a radiação eletromagnética incidente no material. Nesse sentido encontram-se algumas patentes que trata da preparação de blendas contendo polipirrrol (obtido quimicamente), com finalidades antiestáticas e de blindagem eletromagnética. De maneira simplificada, nesses documentos, o método típico de síntese consiste em utilizar um agente oxidante, normalmente $\mathrm{O} \mathrm{FeCl}_{3}$, em polímeros acrílicos ${ }^{[8]}$, resinas de melamina ${ }^{[9]}$, poli(cloreto de vinila) ${ }^{[10]}$, poli(acetato de vinila $^{[11]}$, nylon $^{[12]}$ e poliéster ${ }^{[13]}$ e, posteriormente, essas matrizes são tratadas com pirrol.

Kathirgamanathan $^{[14]}$ preparou MARE aplicando polímeros condutores como adesivo entre camadas de termoplásticos. Já Hourquebie e Olmedo ${ }^{[15]}$ estudaram alguns tipos de blendas de polímeros condutores, polipirrol, politiofenos e polianilina, em diferentes substratos (matrizes poliméricas), para aplicação como MARE. Esses autores verificaram que as propriedades de reflexão da onda incidente pelas blendas de polipirrol/ PVC são similares às de um material magnético convencional, obtidos, por exemplo, pela dispersão do pó de ferrocarbonila em um elastômero. Porém, com um ganho significativo na redução da massa/área. A gramatura da blenda é de $2,6 \mathrm{~kg} \cdot \mathrm{cm}^{-2}$ e a do material magnético convencional é de aproximadamente $5,0 \mathrm{~kg} \cdot \mathrm{cm}^{-2}$.

Desde então, observa-se a tendência de crescimento de estudos envolvendo o comportamento de polímeros condutores como MARE. Mais particularmente considerando o escopo deste estudo, a literatura menciona trabalhos que abordam a avaliação do comportamento do polipirrol na atenuação de micro-ondas pelo uso de diferentes técnicas de preparação do material. Por exemplo, a síntese de polipirrol na presença de ferro carbonila $^{[16]}$, a preparação de nanocompósitos à base de (a) polianilina e polipirrol com ferritas mistas, como a $\mathrm{Zn}_{0,6} \mathrm{Cu}_{0,4} \mathrm{Cr}_{0,5} \mathrm{Fe}_{1,46} \mathrm{Sm}_{0,04} \mathrm{O}_{4}^{[17]}$, (b) polipropileno / montmorilonita / polipirrol ${ }^{[18]}$, (c) $\mathrm{Fe}_{3} \mathrm{O}_{4} /$ polipirrol $^{[19]} \mathrm{e}$ (d) polipirrol com grafeno ${ }^{[20]}$. As propriedades absorvedores de micro-ondas de compostos obtidos pela síntese in situ de polipirrol / Fe-dopado / $\mathrm{TiO}_{2}^{[21]}$ e de filmes de PPi obtidos por diferentes métodos são também avaliadas na área de MARE ${ }^{[22-24]}$.
Nesse sentido, este trabalho busca contribuir na área de MARE à base de polímeros condutores por meio do estudo dos efeitos das condições de preparação do polímero condutor polipirrol via síntese química, pelo uso de dois oxidantes $\left(\mathrm{Fe}_{2}\left(\mathrm{SO}_{4}\right)_{3}\right.$ e $\left.\mathrm{FeCl}_{3}\right)$ e dois surfactantes (dodecilbenzenosulfonato de sódio - DBSNa e ácido dodecilbenzenosulfônico - DBSA). As amostras de PPi obtidas foram caracterizadas por análises de espectroscopia na região do infravermelho (FTIR), difração de raios X (DRX), termogravimetria (TGA), calorimetria exploratória diferencial (DSC) e condutividade elétrica. Blendas de PPi com resina epóxi foram também preparadas e avaliadas como materiais absorvedores de radiação eletromagnética na faixa de 8 a $12 \mathrm{GHz}$.

\section{Experimental}

\section{Materiais}

Os materiais pirrol (Fluka, 97\% pureza), dodecilbenzeno sulfonato de sódio, DBSNa (Fluka), ácido docedilbenzenosulfônico, DBSA (Fluka, 90\% pureza), sulfato de ferro III (Vetec, p.a.), cloreto férrico hexahidratado, $\mathrm{FeCl}_{3} \cdot 6 \mathrm{H}_{2} \mathrm{O}$, (Vetec p.a.) e resina epóxi Araldite®, profissional, disponível comercialmente, foram utilizados sem prévio tratamento.

\section{Síntese química do polipirrol}

O polipirrol foi sintetizado quimicamente utilizando dois tipos de agentes oxidantes $\left(\mathrm{FeCl}_{3}\right.$ e $\left.\mathrm{Fe}_{2}\left(\mathrm{SO}_{4}\right)_{3}\right)$ (Tabela 1). Inicialmente, $0,05 \mathrm{~mol}$ de pirrol foram dissolvidos em $50 \mathrm{~mL}$ de água destilada. Em seguida, esta solução foi adicionada, gota a gota, a duas diferentes soluções de oxidantes: $0,1 \mathrm{~mol}$ de $\mathrm{FeCl}_{3}$ e $0,05 \mathrm{~mol}$ de $\mathrm{Fe}_{2}\left(\mathrm{SO}_{4}\right)_{3}$, respectivamente. Os respectivos meios reacionais foram mantidos sob agitação constante por 4 horas, à temperatura ambiente. Em seguida, o precipitado de PPi obtido foi filtrado e lavado com água destilada. $\mathrm{O}$ pó preto de PPi foi secado em estufa a vácuo a $50{ }^{\circ} \mathrm{C}$ por $16 \mathrm{~h}$.

\section{Síntese química do polipirrol com surfactantes}

O polipirrol foi sintetizado quimicamente por diferentes combinações dos dois oxidantes $\left(\mathrm{FeCl}_{3}\right.$ e $\left.\mathrm{Fe}_{2}\left(\mathrm{SO}_{4}\right)_{3}\right)$ e dos dois surfactantes (DBSNa e DBSA), conforme Tabela 1. Inicialmente, soluções $0,05 \mathrm{~mol}$ de $\mathrm{FeCl}_{3}$ e $0,025 \mathrm{~mol}$ de $\mathrm{Fe}_{2}\left(\mathrm{SO}_{4}\right)_{3}$ foram preparadas em $50 \mathrm{~mL}$ de água destilada. Separadamente, soluções 0,05 mol de DBSNa e DBSA, respectivamente, foram preparadas em $50 \mathrm{~mL}$ de água destilada. As soluções de

Tabela 1. Variação das condições de síntese do PPi.

\begin{tabular}{cccc}
\hline Monômero & Oxidante & Surfactante & Nomenclatura \\
\hline Pirrol & $\mathrm{Fe}_{2}\left(\mathrm{SO}_{4}\right)_{3}$ & DBSNa & PPi-01 \\
Pirrol & $\mathrm{FeCl}_{3}$ & DBSNa & PPi-02 \\
Pirrol & $\mathrm{Fe}_{2}\left(\mathrm{SO}_{4}\right)_{3}$ & DBSA & PPi-03 \\
Pirrol & $\mathrm{FeCl}_{3}$ & DBSA & PPi-04 \\
Pirrol & $\mathrm{Fe}_{2}\left(\mathrm{SO}_{4}\right)_{3}$ & - & $\mathrm{PPi}_{-} \mathrm{SO}$ \\
Pirrol & $\mathrm{FeCl}_{3}$ & - & $\mathrm{PPi}-\mathrm{Cl}$ \\
\hline
\end{tabular}


oxidantes e surfactantes foram misturadas, de acordo com a Tabela 1 e deixadas sob agitação constante por 15 min. Em seguida, 0,08 mol de pirrol foram transferidos para $25 \mathrm{~mL}$ de água destilada e adicionados, gota a gota, na solução de oxidante/surfactante, mantendo-a sob agitação por 4 horas para a polimerização do PPi. Em seguida, o material foi filtrado, lavado e seco em estufa a vácuo por 16 horas.

\section{Preparação das blendas de polipirrol/resina epóxi}

A preparação das blendas PPi/resina epóxi foi realizada variando-se a proporção do polímero condutor (PPi-1; PPi-2; PPi-3; PPi-4; PPi-SO 4 e PPi-Cl) em 1, 10 e $20 \%$, em massa, na resina epóxi (4,0 g). Em seguida, essa mistura foi homogeneizada manualmente por, aproximadamente, 2 minutos e vertida em um molde de alumínio, com 3,0 $\mathrm{mm}$ de espessura. A cura da resina ocorreu em estufa a $60^{\circ} \mathrm{C}$, por 24 horas.

\section{Caracterização}

As amostras de PPi sintetizadas foram inicialmente caracterizadas por espectroscopia de absorção na região do infravermelho com transformada de Fourier (FTIR) pelo uso da técnica de pastilha de $\mathrm{KBr}$, na proporção 0,4:400 mg, utilizando-se um espectrofotômetro Perkin Elmer Spectrum 2000 com transformada de Fourier, na região de $550-4000 \mathrm{~cm}^{-1}$.

A cristalinidade das referidas amostras, com granulometria de $\sim 100$ mesh, foi avaliada utilizando-se um espectrofotômetro de difração de raios $\mathrm{X}$ da marca Philips, modelo PW 1830, com radiação monocromática de $\mathrm{Cu}(\lambda=1,542 \AA)$.

O comportamento térmico dos materiais em estudo foi verificado por análises termogravimétricas (TGA) e de calorimetria exploratória diferencial (DSC), em triplicata, utilizando-se um equipamento da Perkin Elmer, Series Thermal Analysis System, modelo TGA Set e módulo DSC série Pyris, respectivamente. As análises termogravimétricas foram realizadas nas seguintes condições: massa de amostra em torno de $10 \mathrm{mg}$, faixa de temperaturas de 30 a $750{ }^{\circ} \mathrm{C}$, com taxa de aquecimento de $10{ }^{\circ} \mathrm{C} \cdot \mathrm{min}^{-1}$, em atmosferas de ar sintético e nitrogênio, respectivamente. As análises de DSC foram conduzidas conforme os parâmetros a seguir apresentados: massa de amostra $5 \mathrm{mg}$; varredura dinâmica de 30 a $300{ }^{\circ} \mathrm{C}$, com a taxa de aquecimento de $10{ }^{\circ} \mathrm{C} \cdot \mathrm{min}^{-1}$, em atmosfera de nitrogênio e porta-amostra de alumínio hermeticamente fechado.

A condutividade elétrica dos polímeros foi avaliada pelo método de sonda de 4 pontas, baseando-se na literatura ${ }^{[25]}$, com distância entre as pontas de $1,27 \mathrm{~mm}$. Para isto, o material polimérico foi previamente macerado em um almofariz para uniformização do tamanho de partículas. Em seguida, o pó foi prensado em um molde, resultando em pastilhas de PPi com $13 \mathrm{~mm}$ de diâmetro e $1 \mathrm{~mm}$ de espessura. A etapa de prensagem foi realizada com a carga de $5 \mathrm{t}$ por um período de $3 \mathrm{~min}$, para garantir a adequada compactação das pastilhas de PPi. Estas medidas foram realizadas em triplicata, pelo uso de um medidor de condutividade elétrica quatro pontas Cascade
Microtech C4s-64, acoplado a uma fonte Keithley 236, um multímetro e um amperímetro.

As amostras de $\mathrm{PPi} /$ resina epóxi foram analisadas quanto à morfologia pelo uso de um microscópio eletrônico de varredura da marca LEO, modelo 435 Vip, com aumento de 500x. Neste caso, as amostras foram metalizadas com filme de ouro.

A caracterização eletromagnética realizada foi baseada na técnica de medidas de refletividade com placa metálica (placa plana de alumínio). O sistema de medidas utilizado é formado por um acoplador direcional na faixa de 8 a $12 \mathrm{GHz}$, marca Hewlett-Packard, modelo X752C, ligado a um analisador de rede escalar HP8757A e a um gerador de sinais sintetizado 83752A (Agilent), adaptado com cabos coaxiais de baixas perdas, da empresa Adam Russel e Suhner, adaptadores coaxiais de baixas perdas, da empresa Suhner e um microcomputador, com interface GPIB (General Purpose Interface Bus).

\section{Resultados e Discussão}

Os espectros das amostras de $\mathrm{PPi}-\mathrm{Cl}$ e $\mathrm{PPi}-\mathrm{SO}_{4}$, Figura 1, são similares e as principais absorções observadas e suas prováveis atribuições, conforme a literatura $^{[26,27]}$, encontram-se entre 600 e $2000 \mathrm{~cm}^{-1}$. Verificam-se contribuições de vibrações de grupamentos $\mathrm{NH}$ e $\mathrm{OH}$, de acordo com Omastová et al. ${ }^{[28]}$, referentes a vibrações intracadeias. A vibração característica do anel pirrol, o estiramento (v) C-C do anel pirrol, ocorre em $1537 \mathrm{~cm}^{-1}$ para o $\mathrm{PPi}-\mathrm{Cl}$ e se mostra deslocada para maior número de onda $\left(1545 \mathrm{~cm}^{-1}\right)$ para o $\mathrm{PPi}-\mathrm{SO}_{4}$. As bandas em $1451 \mathrm{~cm}^{-1}$ do PPi-Cl e em $1470 \mathrm{~cm}^{-1}$ do $\mathrm{PPi}-\mathrm{SO}_{4}$ correspondem à vibração de $\mathrm{vC}-\mathrm{N}$ no anel. A banda entre $1400-1250 \mathrm{~cm}^{-1}$, que na amostra de $\mathrm{PPi}-\mathrm{Cl}$ apresenta um máximo em $1283 \mathrm{~cm}^{-1}$ e no $\mathrm{PPi}-\mathrm{SO}_{4}$ ocorre em $1296 \mathrm{~cm}^{-1}$, é atribuída à deformação no plano das ligações C-H ou C-N. A região de 1250 a $1100 \mathrm{~cm}^{-1}$, referente à região de vibração do anel pirrólico, mostra pequenas diferenças para as amostras $\mathrm{PPi}-\mathrm{Cl}$ e $\mathrm{PPi}-\mathrm{SO}_{4}$, sendo que seu máximo está situado em $1166 \mathrm{~cm}^{-1}$, para o $\mathrm{PPi}-\mathrm{Cl}$, e em $1178 \mathrm{~cm}^{-1}$ para o $\mathrm{PPi}-\mathrm{SO}_{4}$. Neste caso, está sobreposta à contribuição $\mathrm{vS}=\mathrm{O}$ do ânion sulfato, que absorve na mesma região.

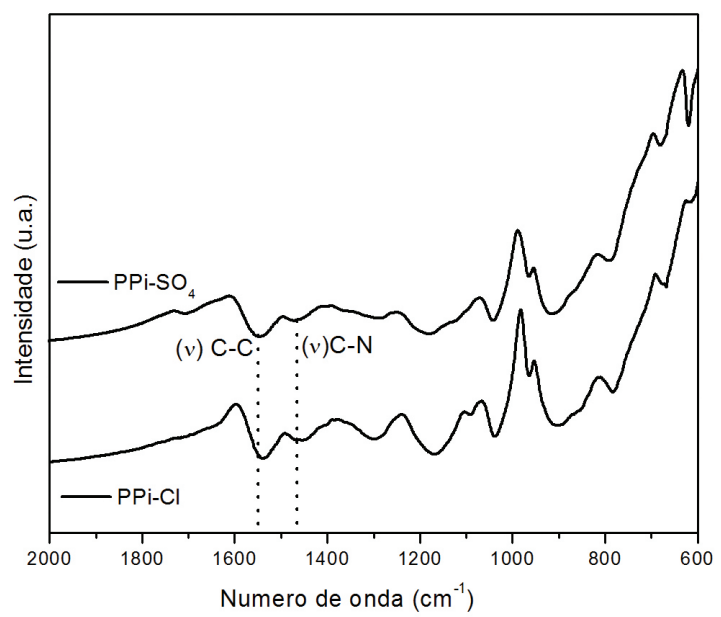

Figura 1. Espectros de FTIR das amostras PPi-Cl e $\mathrm{PPi}_{-} \mathrm{SO}_{4}$ 
Segundo Omastová et al. ${ }^{[28]}$, para o $\mathrm{PPi} \mathrm{SO}_{4}$, a região de deformação no plano das ligações $\mathrm{C}-\mathrm{H}$ e $\mathrm{N}-\mathrm{H}$, em 1130-1000 $\mathrm{cm}^{-1}$ é, provavelmente, influenciada pela vibração $\mathrm{C}-\mathrm{O}$ de unidades hidroxipirrol, formadas pelo ataque nucleofílico da água durante a preparação desta amostra e que, por tautomerismo cetoenólico, acaba produzindo grupos carbonilas, que no espectro $\mathrm{PPi}-\mathrm{SO}_{4}$ não pode ser observado, devido à presença da absorção em $1705 \mathrm{~cm}^{-1}$, região de absorção de grupos carbonila. Outras absorções também são observadas, como bandas atribuídas à vibração de deformação no plano dos grupamentos C-H e N-H, respectivamente em 1037 e $1041 \mathrm{~cm}^{-1}$; e à deformação C-C fora do plano do anel, que ocorre em $963 \mathrm{~cm}^{-1}$, para ambas as amostras. Já a deformação C-H fora do plano do anel ocorre em $912 \mathrm{~cm}^{-1}$ para o $\mathrm{PPi}_{-} \mathrm{SO}_{4}$ e em $895 \mathrm{~cm}^{-1}$ para PPi-Cl. Absorções referentes às deformações $\mathrm{C}-\mathrm{H}$ e $\mathrm{C}-\mathrm{C}$, ambas fora do plano do anel, são também observadas, respectivamente, em 782 e $670 \mathrm{~cm}^{-1}$ para PPi-Cl e em 789 e $679 \mathrm{~cm}^{-1}$ para o $\mathrm{PPi}-\mathrm{SO}_{4}$. Na região de $619-615 \mathrm{~cm}^{-1}$ tem-se a vibração $\mathrm{NH}$ fora do plano, que no espectro $\mathrm{PPi}_{-} \mathrm{SO}_{4}$ está sobreposta por uma absorção do grupo sulfato, que ocorre na mesma região.

A Tabela 2 apresenta as bandas de infravermelho experimental e a comparação com a literatura das amostras de PPi, na faixa de $2000-550 \mathrm{~cm}^{-1[28]}$. As atribuições da literatura são apresentadas entre parênteses.

As amostras de polipirrol preparadas com os oxidantes cloreto férrico ou sulfato férrico, na presença dos surfactantes aniônicos DBSNa ou DBSA, quais sejam, PPi-01 (pirrol $+\mathrm{DBSNa}+\mathrm{Fe}_{2}\left(\mathrm{SO}_{4}\right)_{3}$ ), PPi-02 (pirrol + DBSNa $+\mathrm{FeCl}_{3}$ ), PPi03 (pirrol + DBSA $\left.+\mathrm{Fe}_{2}\left(\mathrm{SO}_{4}\right)_{3}\right)$ e PPi-04 (pirrol $+\mathrm{DBSA}+\mathrm{FeCl}_{3}$ ), apresentam espectros similares. Este comportamento é observado na comparação dos espectros mostrados na Figura 2, que permite afirmar que as amostras PPi-01, PPi-02, PPi-03 e PPi-04 apresentam absorções semelhantes e aparentemente nas mesmas posições. De maneira similar, Omastová et al. ${ }^{[2]}$ ao investigarem polipirróis preparados com os oxidantes cloreto férrico e sulfato férrico, na presença de surfactantes não iônicos, aniônicos e catiônicos, verificaram que as variações observadas referiam-se somente à intensidade dos picos.
A Figura 3 mostra os resultados de difração de raios X. De acordo com a literatura ${ }^{[29,30]}$ o polipirrol é amorfo e o pico em torno de $24^{\circ}$ refere-se à distância intermolecular das cadeias poliméricas. Nas amostras preparadas verifica-se que o padrão de difração se altera em função dos diferentes oxidantes e surfactantes utilizados. Verifica-se que, os ângulos de aparecimento do pico mais intenso para os polímeros $\mathrm{PPi}_{-} \mathrm{SO}_{4}$ e $\mathrm{PPi}-\mathrm{Cl}$ encontram-se em torno de $12^{\circ}$ e $14^{\circ}$, com largura a meia altura de 0,6 e $0,4 \mathrm{~cm}$, respectivamente, Tabela 3 . $\mathrm{O}$ emprego dos surfactantes promoveu o alargamento dos picos e o deslocamento dos mesmos para ângulos maiores, entre $20^{\circ}$ e $22^{\circ}$. Considerando que, maiores ângulos de difração indicam menor distância interplanar, pode-se inferir que há maior interação entre as cadeias poliméricas quando se utiliza os surfactantes. Esta maior proximidade entre as cadeias pode contribuir com maior número de saltos eletrônicos, corroborando com os maiores valores de condutividade elétrica.

A Tabela 3 apresenta os resultados de condutividade elétrica das amostras de PPi. De maneira geral, os polímeros resultantes das sínteses realizadas pelo uso conjunto de oxidante e surfactantes aniônicos apresentam maiores valores de condutividade elétrica $\left(3,3\right.$ a $13{\mathrm{~S} . \mathrm{cm}^{-1}}^{-1}$ ), se comparados com os obtidos para as amostras sintetizadas

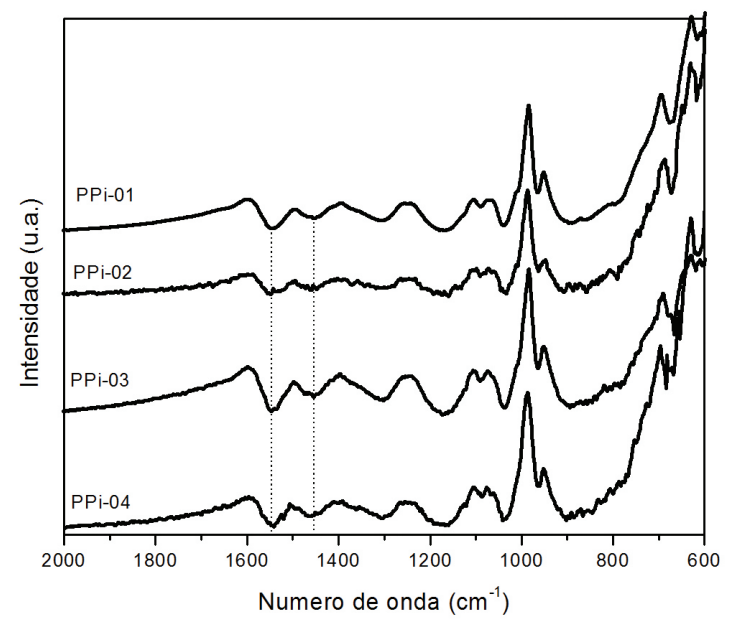

Figura 2. Espectros de FTIR das amostras PPi-01, PPi-02, PPi-03 e PPi-04.

Tabela 2. Contribuições na região do infravermelho das amostras de PPi sintetizadas.

\begin{tabular}{|c|c|c|c|c|c|c|}
\hline $\mathrm{PPi}-\mathrm{SO}_{4}\left(\mathrm{~cm}^{-1}\right)$ & PPi-Cl $\left(\mathrm{cm}^{-1}\right)$ & PPi-01 $\left(\mathrm{cm}^{-1}\right)$ & PPi-02 $\left(\mathrm{cm}^{-1}\right)$ & PPi-03 $\left(\mathrm{cm}^{-1}\right)$ & PPi-04 $\left(\mathrm{cm}^{-1}\right)$ & Atribuição \\
\hline $1545(1552)$ & $1537(1540)$ & 1545 (1548) & 1546 & 1550 & 1544 & (v) $\mathrm{C}-\mathrm{C}$ \\
\hline 1470 (1476) & 1451 (1459) & $1462(1468)$ & 1453 & 1461 & 1452 & $v \mathrm{C}-\mathrm{N}$ \\
\hline 1283 (1289) & 1296 (1308) & $1304(1310)$ & 1304 & - & 1304 & C-H ou C-N \\
\hline 1178 (1197) & 1167 (1166) & 1163 (1183) & 1171 & 1161 & 1167 & $v S=O$ \\
\hline$-(-)$ & 1090 (1094) & 1085 (1094) & 1089 & - & 1089 & $\mathrm{NH}_{2}$ \\
\hline $1041(1050)$ & 1037 (1030) & $1037(-)$ & 1037 & 1035 & 1039 & $\mathrm{C}-\mathrm{H}$ e N-H \\
\hline $963(-)$ & 963 (967) & $962(-)$ & 963 & - & 964 & C-C \\
\hline $912(920)$ & 897 (915) & 899 (915) & 891 & 907 & 888 & $\mathrm{C}-\mathrm{H}$ \\
\hline 789 (796) & 782 (796) & $-(-)$ & - & - & - & $\mathrm{C}-\mathrm{H}$ \\
\hline $679(678)$ & $670(678)$ & $670(678)$ & 667 & 672 & 672 & $\mathrm{C}-\mathrm{C}$ \\
\hline $619(620)$ & $615(-)$ & $612(-)$ & 603 & 614 & 614 & $\mathrm{NH}$ \\
\hline
\end{tabular}

As atribuições entre parênteses correspondem a dados da literatura ${ }^{[28]}$. 


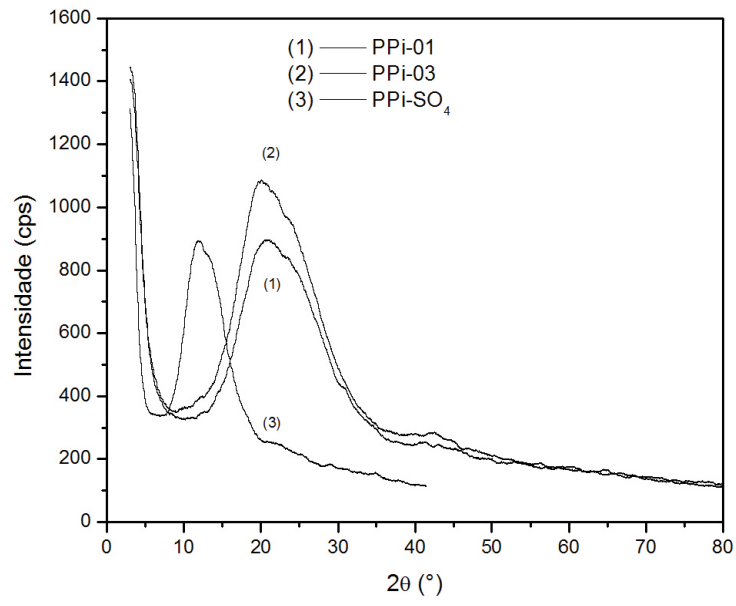

(a)

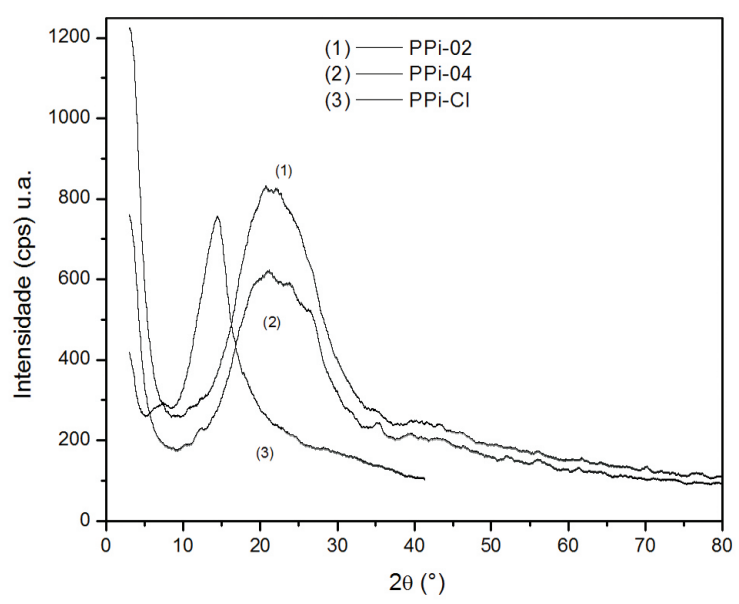

(b)

Figura 3. DRX das amostras de (a) $\mathrm{PPi}_{-} \mathrm{SO}_{4}$ e (b) PPi-Cl sem e com os surfactantes DBSNa e DBSA.

Tabela 3. Condutividade elétrica $(\sigma)$, ângulos dos picos mais intensos $(2 \theta)$, distância interplanar, largura a meia altura $\left(\mathrm{L}_{1 / 2}\right)$ e tamanho dos cristalitos (D), obtidos por DRX, das amostras de PPi estudadas.

\begin{tabular}{cccccc}
\hline Amostras & $\sigma$ & $\mathbf{2 \theta}$ & $\begin{array}{c}\text { Distância } \\
\text { interplanar }(\mathbf{A})\end{array}$ & $\mathbf{L}_{\mathbf{1} / 2}(\mathbf{c m})$ & $\mathbf{D}(\mathbf{n m})$ \\
\hline PPi-SO & & 12 & 7,369 & 0,6 & 230 \\
PPi-Cl & $0,28 \pm 0,001$ & 14 & 6,320 & 0,4 & 345 \\
PPi-01 & $0,13 \pm 0,002$ & 21 & 4,227 & 1,1 & 126 \\
PPi-02 & $13 \pm 2,3$ & 20 & 4,436 & 1,1 & 126 \\
PPi-03 & $3,3 \pm 0,003$ & 21 & 4,227 & 1,1 & 127 \\
PPi-04 & $8,9 \pm 0,05$ & 22 & 4,037 & 1,3 & 108 \\
\hline
\end{tabular}

somente pelo uso dos oxidantes $\left(0,13\right.$ e $0,28{\left.\mathrm{~S} . \mathrm{cm}^{-1}\right)}^{-}$ Verifica-se, também, que as amostras preparadas pelo uso do oxidante $\mathrm{Fe}_{2}\left(\mathrm{SO}_{4}\right)_{3}$ mostram valores de condutividade elétrica mais elevados, em comparação às obtidas pelo uso do oxidante clorado, incluindo as modificadas pelo uso de surfactantes (PPi-01 e PPi-03), que se apresentam ainda mais condutoras (13 e 8,9 S.cm-1, respectivamente). Esses resultados mostram que o uso do surfactante promove o aumento da condutividade elétrica em uma ordem de grandeza. Conforme discutido anteriormente nas análises de difração de raios $X$, este aumento de condutividade pode estar relacionado com a proximidade dos centros ativos, que promovem os saltos eletrônicos. Além disso, segundo a literatura ${ }^{[29,30]}$, o surfactante aniônico atua durante a polimerização como uma estrutura codopante, o que contribui para o aumento da condutividade elétrica do polímero.

Em paralelo, verifica-se que essas contribuições estão associadas a menores tamanhos de cristalitos (valores D determinados a partir da equação de Scherrer), Tabela 3. De acordo com a literatura, o menor tamanho dos cristalitos de um material aumenta o número de interfaces e de contatos entre os mesmos, com a predominância das conduções eletrônica e iônica ${ }^{[31]}$.

Os comportamentos térmicos destes materiais foram avaliados por termogravimetria e calorimetria exploratória diferencial, Figuras 4-6. A Figura 4 mostra as curvas TGA de amostras de $\mathrm{PPi}_{-} \mathrm{SO}_{4}$ e PPi-Cl, obtidas em atmosferas de ar sintético e nitrogênio. Observa-se que, a atmosfera do forno não influencia o comportamento térmico das amostras de PPi. Verificam-se, para ambas as atmosferas, duas etapas de perda de massa. A primeira etapa, entre 30 e $100{ }^{\circ} \mathrm{C}$, atribuída à perda de água residual contida na estrutura do PPi. A segunda etapa correspondente à degradação da cadeia polimérica inicia-se em torno de $190{ }^{\circ} \mathrm{C}$. Este valor de temperatura de início de degradação é favorável para o processamento de absorvedores de uso aeronáutico, pois atende aos requisitos deste setor, que necessita de materiais estáveis até $120{ }^{\circ} \mathrm{C}^{[32]}$. Adicionalmente, verifica-se uma etapa adicional de perda de massa, entre 190 a $250{ }^{\circ} \mathrm{C}$, para a amostra de PPi-SO atribuída à evolução do $\mathrm{SO}_{2}$, conforme também observado na literatura ${ }^{[29,30]}$. Considerando-se que, o possível uso desse material no setor aeronáutico é em atmosfera normal de oxigênio, todas as outras análises foram realizadas em atmosfera de ar.

Observa-se claramente que a adição de surfactante durante a síntese influencia o comportamento térmico das amostras de PPi, Figura 5. A Figura 5b refere-se à ampliação da região inicial (30\% perda de massa) da Figura 5a. A temperatura de início de degradação da cadeia polimérica é similar ao observado para as amostras de $\mathrm{PPi}-\mathrm{Cl}$ e PPi-SO $\mathrm{P}_{4}$, sem a adição de surfactante (Figura 4). Porém, após essa primeira etapa, verifica-se a presença de mais etapas de termodecomposição. Baseando-se em dados da literatura ${ }^{[33]}$, pode-se considerar que ocorre, 


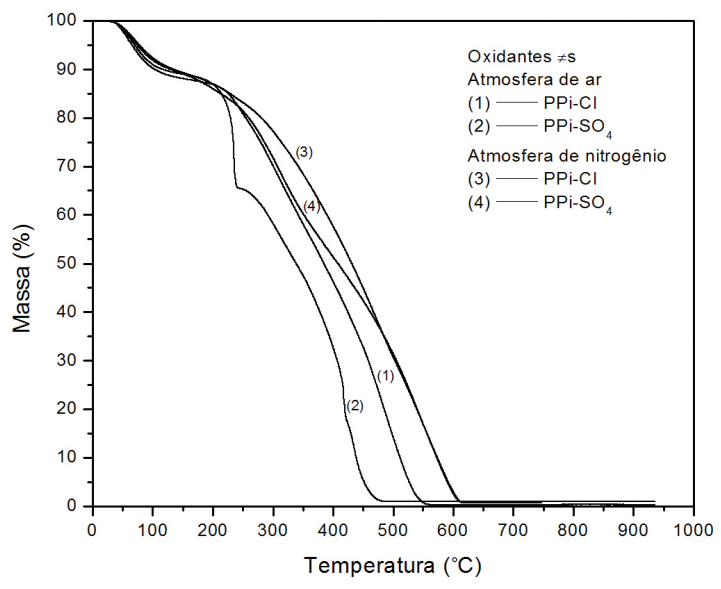

Figura 4. Curvas TGA das amostras de $\mathrm{PPi}-\mathrm{Cl}$ e $\mathrm{PPi}_{-} \mathrm{SO}_{4}$ em atmosferas de ar e de nitrogênio.

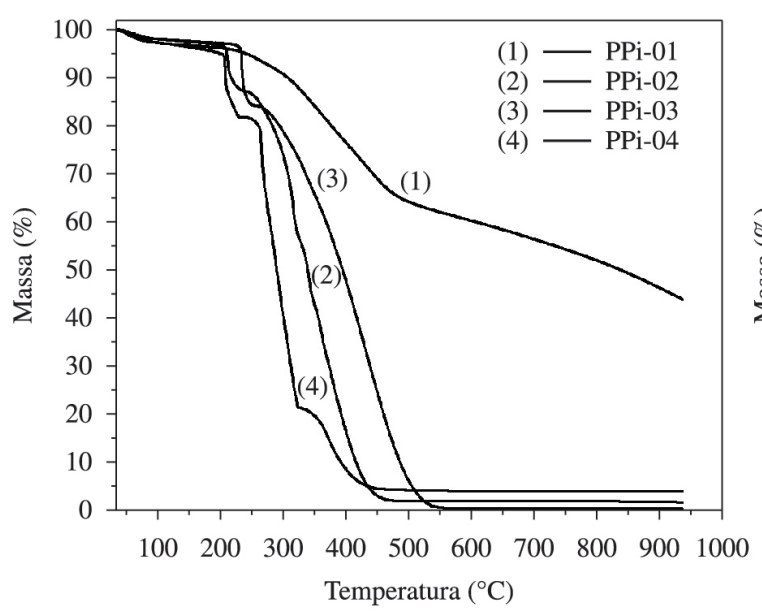

(a) inicialmente, a cisão da ligação $\mathrm{PPi}_{-} \mathrm{SO}_{4}$ ou $\mathrm{PPi}-\mathrm{Cl}$ $\left(\sim 215-260{ }^{\circ} \mathrm{C}\right)$, com a volatilização do surfactante.

É interessante notar que, a amostra PPi-01 (PPi$\mathrm{SO}_{4}$-DBSNa) apresenta comportamento térmico distinto das outras amostras, com maior estabilidade térmica, devido à perda de massa ocorrer de maneira mais suave, além de apresentar um maior resíduo no final da análise, em torno $45 \%$, próximo da temperatura de $950{ }^{\circ} \mathrm{C}$. Este comportamento sugere que o polímero PPi-01 possui maior interação entre os componentes da cadeia polimérica ou uma possível maior massa molar. As amostras PPi-02, PPi-03 e PPi-04 mostram comportamentos de degradação térmica semelhantes, com o início de perda de massa em torno de $200{ }^{\circ} \mathrm{C}$ e um aumento brusco na termodegradação, até a quase completa degradação do polímero ( $0 \%$ de resíduo), em torno de $450{ }^{\circ} \mathrm{C}$.

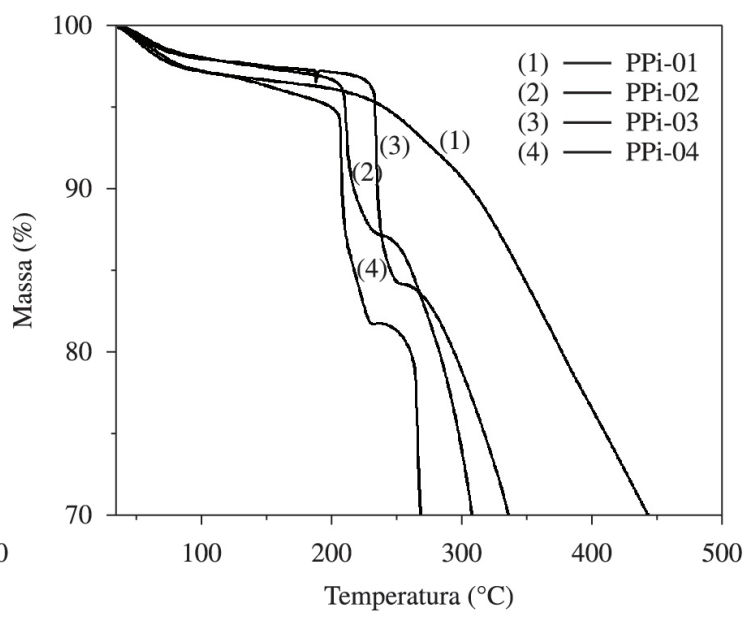

(b)

Figura 5. Curvas TGA do PPi com diferentes oxidantes e surfactantes (a) e ampliação das curvas, na região de início da degradação (b), em atmosfera de ar.

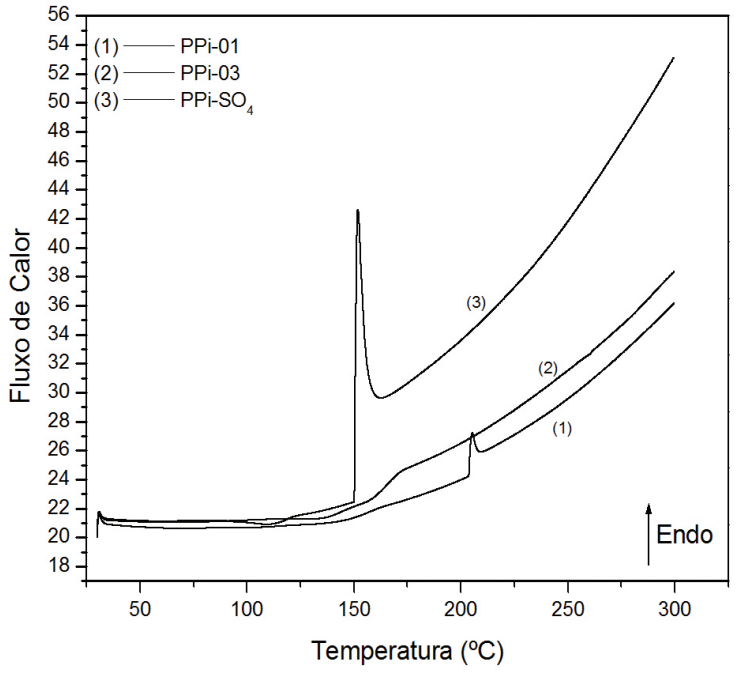

(a)

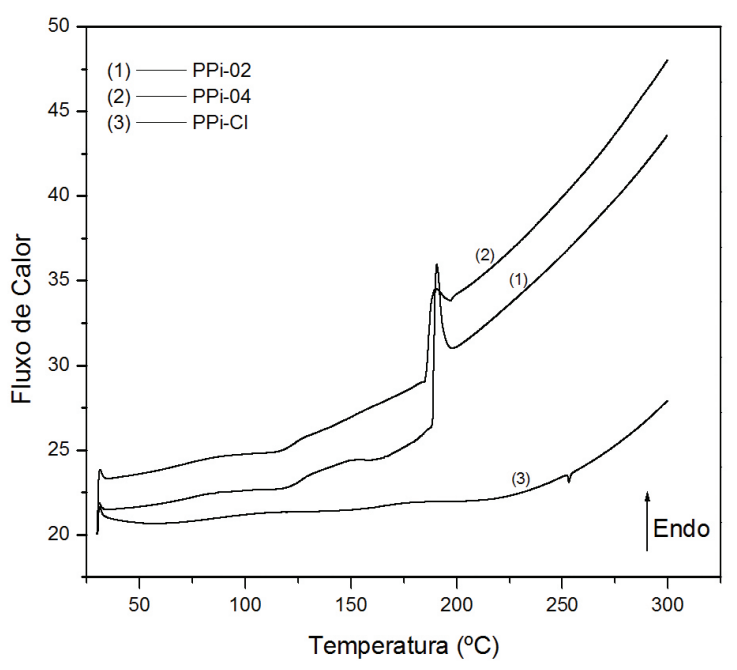

(b)

Figura 6. Curvas de DSC de (a) $\mathrm{PPi}_{-} \mathrm{SO}_{4}$ e (b) PPi-Cl com e sem os surfactantes DBSNa e DBSA, em atmosfera de ar. 
A Figura 6 é representativa das curvas de DSC obtidas em varredura dinâmica das amostras de PPi, na faixa de temperaturas de 30 a $350{ }^{\circ} \mathrm{C}$, em portaamostras de alumínio fechados. Observa-se que, nesta condição, as curvas das amostras de PPi não apresentam pico endotérmico na região de $50-120{ }^{\circ} \mathrm{C}$, referente à eliminação de água, como mostram as análises termogravimétricas. Verifica-se a presença de um pico endotérmico intenso $\left(\sim 152{ }^{\circ} \mathrm{C}\right.$, Figura 6a) para a amostra $\mathrm{PPi}-\mathrm{SO}_{4}$, concordante com a perda de massa observada na análise termogravimétrica (Figura 4), referente à degradação térmica. As curvas de DSC também mostram picos endotérmicos nas temperaturas de 205, 190 e $191{ }^{\circ} \mathrm{C}$, para as amostras $\mathrm{PPi}_{-} \mathrm{SO}_{4}-\mathrm{DBSNa}, \mathrm{PPi}-\mathrm{Cl}-$ DBSNa e PPi-Cl-DBSA, respectivamente (Figuras 6a, b). Estes picos são atribuídos a diferentes eventos, que, geralmente, ocorrem nesta faixa de temperatura, como a presença de estabilizantes e/ou solventes residuais do

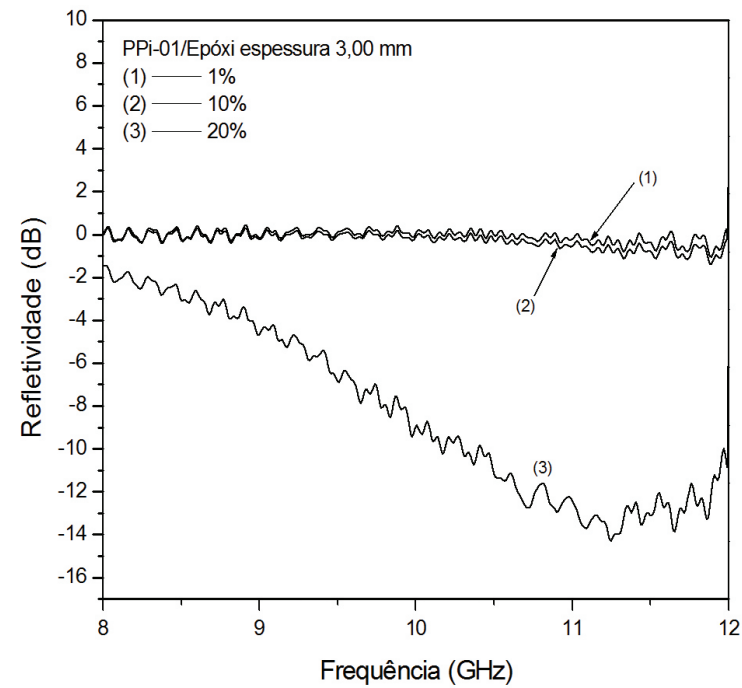

(a)

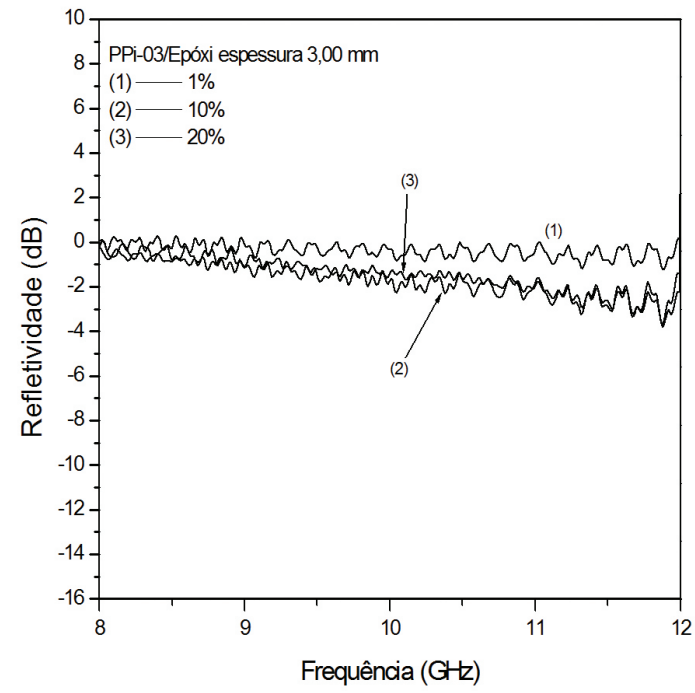

(c) monômero pirrol. A correlação destes resultados com as análises termogravimétricas realizadas permite afirmar que os eventos endotérmicos registrados referem-se à degradação do complexo polímero-oxidante ou polímerooxidante-surfactante.

Como já observado nas análises de TGA, as análises de DSC mostram a influência dos surfactantes nos comportamentos térmicos dos PPi sintetizados. Valores de Tg em 160, 170, 124 e $126^{\circ} \mathrm{C}$ para as amostras PPi01,PPi-03, PPi-04 e PPi-02, respectivamente. Para a amostra $\mathrm{PPi}-\mathrm{SO}_{4}$, a presença desse evento é mascarada pelo pico que ocorre nessa região e não é possível observar para a amostra PPi-Cl. Verifica-se maiores valores de transição vítrea para amostras preparadas com o oxidante $\mathrm{Fe}_{2}\left(\mathrm{SO}_{4}\right)_{3}$, independentemente do surfactante utilizado, o que corrobora com os comportamentos térmicos apresentados nas curvas termogravimétricas, sugerindo, mais uma vez, que estas amostras apresentam uma

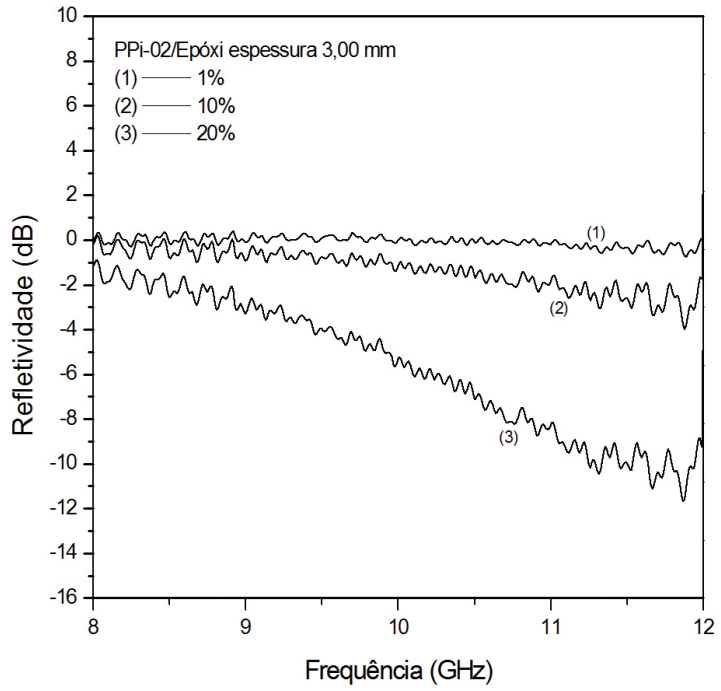

(b)

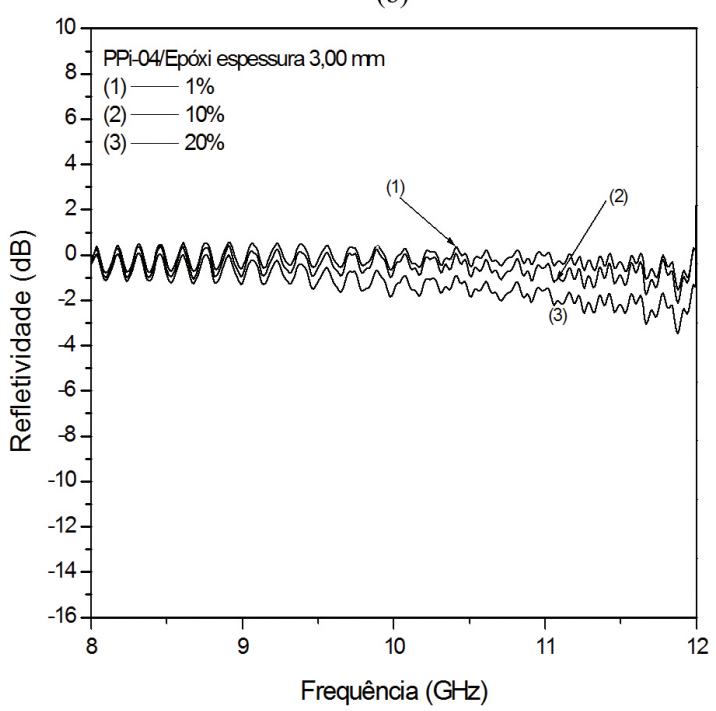

(d)

Figura 7. Curvas de refletividade em função da frequência para as blendas de PPi/resina epóxi, com 3,0 mm de espessura. (a) $\mathrm{PPi}^{-\mathrm{SO}_{4}}$ DBSNa (PPi-01), (b) PPi-Cl-DBSNa (PPi-02), (c) PPi-SO -DBSA (PPi-03) e (d) PPi-Cl-DBSA (PPi-04). 


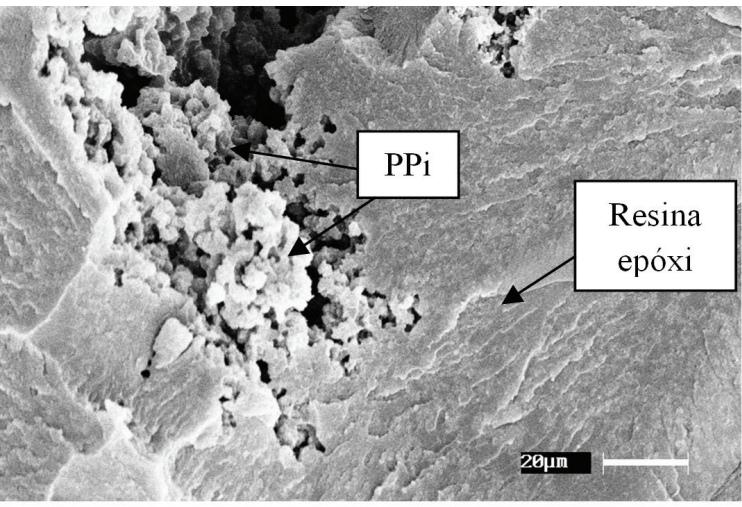

(a)

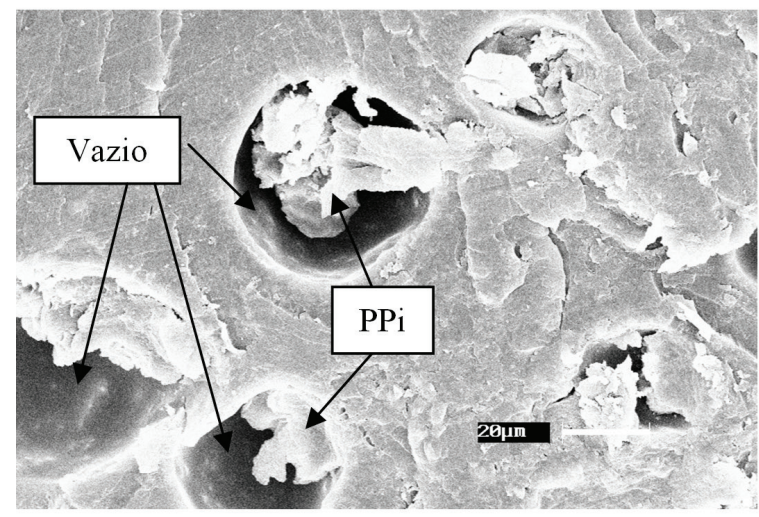

(c)

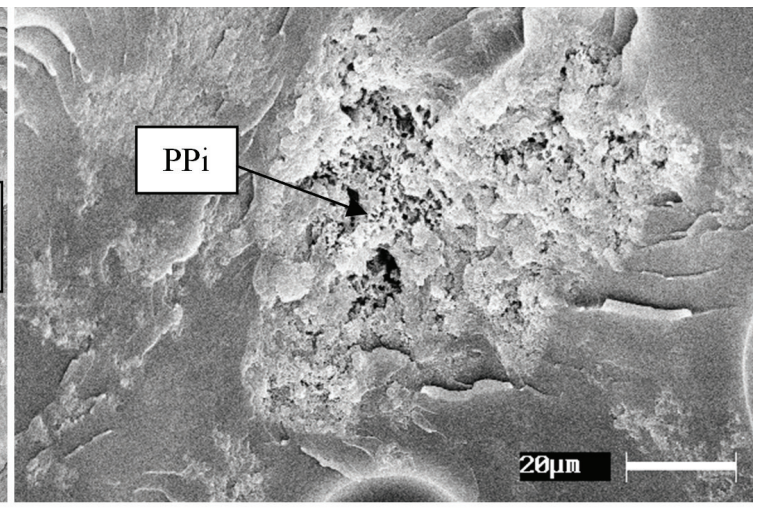

(b)

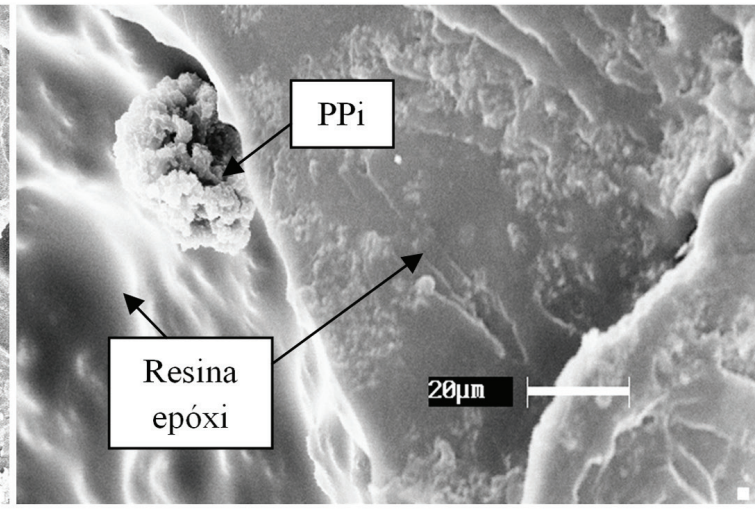

(d)

Figura 8. Microscopias eletrônicas de varredura das blendas de PPi/resina epóxi, com 20\% em massa de PPi. (a) $\mathrm{PPi}_{-} \mathrm{SO}_{4}-\mathrm{DBSNa}$ (PPi-01), (b) PPi-Cl-DBSNa (PPi-02), (c) PPi-SO - -DBSA (PPi-03) e (d) PPi-Cl-DBSA (PPi-04).

possível fração de maior massa molar. De acordo com a literatura $^{[25,34]}$, a temperatura de transição vítrea do PPi ocorre ao redor de $350{ }^{\circ} \mathrm{C}$, considerando-se, no entanto, que esse valor fortemente dependente da técnica utilizada na obtenção do polímero (química, eletroquímica, fotoquímica), como também das condições de preparação, envolvendo o fator de pureza do monômero, o qual depende da procedência deste reagente.

A Figura 7 apresenta as curvas de refletividade das blendas dos polímeros $\mathrm{PPi}-\mathrm{SO}_{4}-\mathrm{DBSNa}, \mathrm{PPi}-\mathrm{Cl}-\mathrm{DBSNa}$, PPi-SO $\mathrm{S}_{4}$-DBSA e PPi-Cl-DBSA com resina epóxi, na espessura de 3,0 mm. Comparando-se as Figuras 7a-d observa-se, inicialmente, os melhores desempenhos na atenuação para os materiais processados com as maiores concentrações de polipirrol (20\% em massa) na resina epóxi, ou seja, os valores de refletividade diminuem (escala negativa) progressivamente, com o aumento do PPi na resina epóxi. E, nesse sentido, verifica-se ainda que os melhores valores de atenuação são observados para as amostras PPi-01 (PPi-SO - DBSNa) e PPi-02 (PPi- Cl-DBSNa), Figuras 7a, b, com valores de $-15 \mathrm{~dB}$ e $-13 \mathrm{~dB}$, na frequência de $11,5 \mathrm{GHz}$, respectivamente, que correspondem a valores de absorção de, aproximadamente, $95 \%$. Os resultados de refletividade obtidos sugerem que o surfactante DBSNa tem efeito preponderante neste comportamento, para esta faixa de frequências de medidas. Este efeito está relacionado com a dispersão das partículas na matriz, considerando que quanto menores e mais homogeneamente dispersas na matriz, mais bem estabelecida fica a formação de caminhos de condução interpartículas, favorecendo, assim, o desempenho como centros absorvedores de radiação eletromagnética.

A Figura 8 apresenta micrografias obtidas por microscopia eletrônica de varredura, representativas das blendas dos polímeros $\mathrm{PPi}-\mathrm{SO}_{4}$-DBSNa, PPi-Cl-DBSNa, PPi-SO $\mathrm{SO}_{4}$-DBSA e PPi-Cl-DBSA em resina epóxi, contendo $20 \%$ em massa de PPi. As imagens mostram que a blenda preparada com o PPi dopado com o surfactante DBSNa (Figuras 8a, b) apresentam regiões mais extensas de grânulos de PPi, ou seja, o polímero condutor está melhor distribuído e com melhor interação com a matriz de resina epóxi. Em contrapartida, as imagens representativas das blendas de PPi-DBSA (Figuras 8c, d) apresentam regiões de PPi mais compactadas, onde o aglomerado de polímero apresenta-se inserido em um poro.

Os resultados de refletividade e de microscopia eletrônica de varredura são concordantes com os comportamentos observados nas determinações de difração de raios $\mathrm{X}$ e de condutividade elétrica, como mostra a Tabela 3.

\section{Conclusão}

Polipirrol com condutividades na faixa de 0,1 a $13 \mathrm{~S} / \mathrm{cm}$ foi preparado com o auxílio de oxidantes $\left(\mathrm{FeCl}_{3}\right.$ e $\left.\mathrm{FeSO}_{4}\right)$ e surfactantes (DBSA e DBSNa). 
Análises estruturais por espectroscopia na região do infravermelho mostram que não há alterações significativas nos espectros, indicando que as amostras de polímeros obtidas apresentam estruturas químicas similares. Entretanto, a organização no estado sólido é influenciada pelos surfactantes obtendo-se materiais com maior ordenamento estrutural. Além disso, polipirróis sintetizados na presença de surfactantes apresentam maiores valores de degradação térmica, principalmente àqueles preparados na presença de $\mathrm{SO}_{4}^{2-}$ e DBSNa. Adicionalmente, o ânion sulfato, independentemente do surfactante apresenta maiores valores de condutividade elétrica (PPi-SO - -DBSNa (13 S.cm-1) e PPi-SO - DBSA

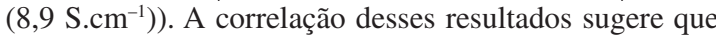
o uso do par oxidante-surfactante favoreceu o aumento da fração molar do polímero. A preparação de blendas de PPi com resina epóxi resultou em materiais com propriedades absorvedoras de radiação eletromagnética. Blendas contendo $20 \%$ em massa de $\mathrm{PPi}-\mathrm{SO}_{4}$-DBSNa apresentam valores de $95 \%$ de absorção da radiação incidente em $11,5 \mathrm{GHz}$, sugerindo que esses materiais são promissores como centro absorvedores de radiação eletromagnética.

\section{Agradecimentos}

As autoras agradecem ao $\mathrm{CNPq}$ (142314/2010-2 e 305478/2009-5), à Dra. Miriam Kasumi Hwang da Divisão de Materiais do Instituto de Aeronáutica e Espaço pelo apoio na análise dos dados de difração de raios $\mathrm{X}$ e às Divisões de Materiais e de Química do Instituto de Aeronáutica e Espaço pelo apoio na realização deste estudo.

\section{Referências Bibliográficas}

1. Angelopoulos, M. - J. Res. Dev., 45, p.57 (2001).

2. Beaujuge, P. M. \& Reynolds, J. R. - Chem. Rev., 110, p.268 (2010). PMid:20070115. http://dx.doi.org/10.1021/ cr900129a

3. Dall'Olio, A.Cascola, Y.; Varacca, V. \& Bocchi, V. - Comptes Rendus, 267, p.433 (1968)

4. Waltman, R. J. \& Bargon, J. - Can. J. Chem., 64, p.76 (1986). http://dx.doi.org/10.1139/v86-015

5. Diaz, A. F.; Castilho, J. I.; Logan, J. A. \& Lee, W. Y. - J. Electroanal Chem., 129, p.115 (1981). http://dx.doi. org/10.1016/S0022-0728(81)80008-3

6. Bocchi, V. \& Gardini, G. P. - J. Chem. Society, Chem. Commun., 2, p.148 (1986). http://dx.doi.org/10.1039/ c3986000148a

7. Omastová, M.; Trchová, M.; Kovárová, J. \& Stejskal, J. - Synth. Metals, 138, p.447 (2003). http://dx.doi. org/10.1016/S0379-6779(02)00498-8

8. Osawa, T.; Yoshino, K. \& Kanefuji, K. - Chem. Abstr., 106, p.P85923h (1987).

9. Naarmann, H.; Krueckau, F. E.; Moebius, K. H. \& Ott, K. - Chem. Abstr., 110, p.P184261e (1989).

10. Kaneko, T.; Suzuki, H.; Matsui, N. \& Katoh, T. - Chem. Abstr., 119, p.P73842m (1993)

11. Myata, S. - Chem. Abstr., 119, p.P205616c (1993).

12. Mizoguchi, I. - Chem. Abstr., 116, p.P85768t (1992).

13. Mizoguchi, I. - Chem. Abstr.,116, p.P246314j (1992).
14. Kathirgamanathan, P. - Polymer, 34, p.3105 (1993). http:// dx.doi.org/10.1016/0032-3861(93)90641-M

15. Hourquebie, P. \& Olmedo, L. - Synth. Met., 65, p.19 (1994). http://dx.doi.org/10.1016/0379-6779(94)90288-7

16. Li, D.; Wang, H.; Zhao, J. \& Yang, X. - Mat. Chem. Physics, 130, p.437 (2011). http://dx.doi.org/10.1016/j. matchemphys.2011.07.005

17. Li, L.; Xiang, C.; Liang, X. \& Hao, B. - Synth. Metals, 160, p.28 (2010). http://dx.doi.org/10.1016/j. synthmet.2009.09.026

18. Moucka, R.; Mravcáková, M.; Vilcaková, J.; Omastová, M. \& Sáha, P. - Mat. Design, 32, p.2006 (2011).

19. Li, Y.; Chen, G.; Li, Q.; Qiu, G. \& Liu, X. - J. Alloys Comp., 509, p.4104 (2011). http://dx.doi.org/10.1016/j. jallcom.2010.12.100

20. Liu, P.; Huang, Y.; Wang, L. \& Zhang, W. - J. Alloys Comp., 573, p.151 (2013). http://dx.doi.org/10.1016/j. jallcom.2013.03.280

21. Li, Q.; Zhang, C. \& Li, J. - J. Alloys Comp., 509, p.1953 (2011). http://dx.doi.org/10.1016/j.jallcom.2010.10.099

22. Hakansson, E.; Amiet, A.; Nahavandi, S. \& Kaynak, A. - European Pol. J., 43, p.205 (2007).

23. Kaynak, A.; Hakansson, E. \& Amiet, A. - Synth Metals, 159, p.1373 (2009). http://dx.doi.org/10.1016/j. synthmet.2009.03.013

24. Jamadade, S.; Jadhav, S. V. \& Puri, V. - J. Non-Cryst. Solids, 357, p.1177 (2011).

25. Girotto, E. M. \& Santos, I. A. - Quím. Nova, 25, p.639 (2002).

26. Smith, A. L. - "Applied Infrared Spectroscopy", John Wiley \& Sons, New York (1979).

27. Silverstein, R. M.; Bassler, G. C. \& Morrill, T. C. - "Spectrometric Identification of Organic Compounds", John Wiley \& Sons, New York (1981).

28. Omastová, M.; Trchová, M.; Pionteck, J.; Prokes, J. \& Stejskal, J. - Synth. Metals, 143, p.153 (2004). http:// dx.doi.org/10.1016/j.synthmet.2003.11.005

29. Kassim, A.; Basar, Z. B. \& Mahmud, H. N. M. E. - Proc. Indian Acad. Sci., 114, p.152 (2002).

30. Omastová, M. \& Pionteck, J.; Trchová, M. - Synth. Metals, 135-136, p.437 (2003). http://dx.doi.org/10.1016/ S0379-6779(02)00582-9

31. Muccillo, E. N. S.; Porfírio, T. C.; Tadokoro, S. K.; Rey, J. F. Q.; Rocha, R. A.; Steil, M. C. \& Muccillo, R. - Cerâmica, 51, p.157 (2005). http://dx.doi.org/10.1590/ S0366-69132005000200014

32. Folgueras, L. C., Alves, M. A. \& Rezende, M. C. - J. Aerosp. Techn. Manag., 2, p.63 (2010). http://dx.doi.org/10.5028/ jatm.2010.02016370

33. Jakab, E.; Mészáros, E. \& Omastová, M. - J. Therm. Anal. Cal., 88, p.515 (2007). http://dx.doi.org/10.1007/s10973006-8241-7

34. Ultrscki, L. A. - "Polymer Alloys and Blends. Thermodynamics and reology", Hanser, New York (1989). 